\title{
Analysis of the Analytical and Simulations Results with the Network Model and Dynamic Priority of Limited Buffer
}

\author{
Fathollah Dadgar Arablou \\ Baku state University, AZ 1148, Baku, \\ Av. Academy of $Z$. Khalilova
}

\author{
Aliyev Alakbar \\ Baku State University, Az 1148,Baku, \\ Av,Academy of Z.Khalilova
}

\begin{abstract}
The problem of choosing optimal parameters for dynamic priorities in the case of the various types of service requests with linearly decreasing function of priorities, The results of analysis and simulations models of distributed computer networks with dynamic priorities and a limited buffer residence time requirements of the network is limited. The network constraint violation results in a loss requirements. A comparative analysis of the results of analytical and simulation models are conducted to verify the adequacy of the analytical models.
\end{abstract}

\section{General Terms}

Service, limited buffer, dynamic priorities, network

\section{Keywords}

Service, dynamic priorities, Optimization, Dynamic priorities, Priority

\section{INTRODUCTION}

The process of managing distributed computer networks with dynamic priorities, requirements and limited buffer. Such services are used in the service system, in which subscribers are in moving vehicles. Under the service is understood pretreatment coordinate information and vehicle speed. The time between the arrival of the requirements of the system and the end of the service is limited to a certain value, and if this time exceeds this value, the requirements will be lost. This information received too late for the consumer, so it is useless [1-3].In [4] studied the process of managing distributed networks with dynamic priorities, requirements and limitations of the buffer is shown that the lower limit of the total losses reached at the final value of the queue length. Using these results solved the problem of determining the optimal characteristics of the service process in a distributed computer networks, and often it is to some extent complicates the operating system.However, the development of more advanced algorithms for the organization of the service process are able to reduce the loss of requirements, accompanied by the growth performance of the network services. A special feature of this approach is to minimize the loss of claims due to the preventive removal of some of them, without waiting for the service.A feature of these networks is to minimize losses due to the requirements of preventive removal of some of them, without waiting for the service. In order to study the functioning of such a network, it can be considered as a model of a queuing system (QS). It is necessary to solve the problem of optimization of parameters determining the nature of the preventive removal requirements, resulting in minimizing their losses. In order to confirm the adequacy of the analytical models developed in [5], the need to develop simulation models of such networks and services according to certain criteria to compare the results of both models. As it is known to meet specified levels of quality of service (Quality of Service) in switches highspeed multimedia networks with a total buffer space consisting of (Shared Buffering) with a number of spatial priorities (Space Priorities), objectified making procedures in different types of applications uses a buffer of time priority (Time priorities), defining the rules for selecting the type and appearance of the s buffer [6]. Thus in the classical scheme of priority service, it is assumed that a certain type of application compared to other bids at Go type have a higher priority simultaneously both species. The analysis shows that known in a very small amount of work, the cat on the ryh considered schemes with different levels of spatial priorities for sound control in lyayuschie intensities (probably) different types of applications, and loss of time, etc., and priorities affecting the time of their $s$ and delay (latency) in the buffer. In [7-8] proposed various schemes of spatial definition of priorities in order to find the optimal (in some sense) the size used in Buffer Memory in the nodes of different service networks. In [9-10] based on the actual working conditions of the first high-speed multimedia service networks as temporary priority is proposed to use the dynamic priorities change over time.

\section{SOLUTION OF THE PROPBLEM}

The input stage of the QS (Fig. 1) enters the flow requirements. Because of the requirements of the queue defined by some rule, come with equal probability for all serviceable and free from computer maintenance.

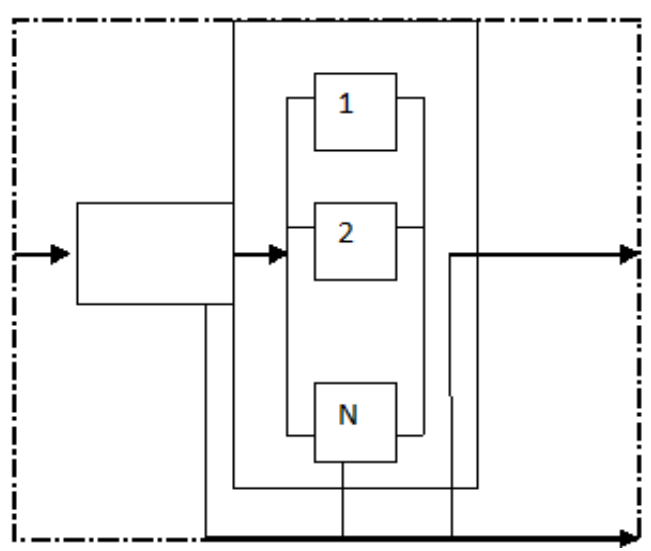

Fig.1. QS Limited queue

After leaving the service requirements of the system. Services suggest a uniform input stream with equal claims priori priorities. In the process of dynamic priority service requirements may vary depending on the situation. The service time is distributed over all the requirements of the same Rule. The network operation is possible if the situation 
changes in two places: in the queue and service. Stay requirements system consists of two phases: waiting and service, and the total residence time in the system requirements should not exceed $\tau_{s}^{*}$. On standby phase requirement may be removed from the system according to some rule. If newcomers requirement finds a busy queue, the first requirement of the queue (the "old") is deleted as it is more than all the others in the queue does not have a chance to meet the time limit for staying in the $\mathcal{\tau}_{\boldsymbol{s}} \leq \boldsymbol{\tau}_{\boldsymbol{s}}^{*}$, the remainder of the queue are moved up one position forward, and again came in last place. Such requirements make the loss of the first kind $\boldsymbol{D}_{\mathbf{1}}$ - In the maintenance phase (or immediately after its completion) requirement can be removed from the system, if it is the residence time in the

$\left(\mathcal{\tau}_{\boldsymbol{s}}\right)$ exceeds $\tau_{s}^{*}$. These requirements are the losses of the second kind $\boldsymbol{D}_{2}$. The aim of solving the problem is the choice of such a rule and served awaiting removal requirements, in which the expectation value of the total losses of both genders was minimal:

$$
\begin{aligned}
& M\left[P\left(p_{1}, p_{2}\right)\right] \longrightarrow \min \\
& L_{q} \leq m \tau_{s} \leq \tau_{s}^{*}
\end{aligned}
$$

It is assumed that requirements are placed in the queue in order of arrival, i.e. removed will always be a requirement in the queue first. The system places the number of queuing is limited to $\mathrm{m}$.

Note that the optimal organization of the rules of service makes it possible to get the maximum efficiency of the system by removing from the system before or during maintenance of those requirements which do not fulfill the condition of $\mathcal{\tau}_{\boldsymbol{s}} \leq \mathcal{\tau}_{\boldsymbol{s}}^{*}$. For an exponential service time value $\sum_{\boldsymbol{q}}$ can be defined as follows [4]:

$$
\boldsymbol{L}_{q}=P \boldsymbol{P} /(\mathrm{N}-\boldsymbol{P})_{\text {In this }}
$$

formula, depending on the nature of the object, you can use the system to allow the following approximations:

$$
\begin{aligned}
& \text { In } \rho<<1 \quad L_{q} \longrightarrow \rho^{N+1} / N^{2} \\
& { }_{\text {In }} \lambda / \mu N \rightarrow 1 \quad L_{q} \rightarrow \rho /(N-\rho)
\end{aligned}
$$

\section{$\sum_{\boldsymbol{q}}$ can be determined known as the waiting time in the}

queue $\mathcal{\mathcal { T }}_{\boldsymbol{q}}$ requirements, the residence time requirements in the $\mathcal{Z}_{\boldsymbol{s}}$, the expected number of claims in the $\boldsymbol{L}_{\boldsymbol{s}}$ :

$$
\begin{aligned}
& \tau_{q}=\boldsymbol{L}_{\boldsymbol{q}} / \lambda \\
& \boldsymbol{\tau}_{s}=\boldsymbol{L}_{s} / \lambda \\
& \boldsymbol{L}_{s}=\boldsymbol{L}_{q}+\rho
\end{aligned}
$$

Because the maintenance organization in the system queue is limited, and determining a value of the residence time requirements of the system is also very difficult, even impossible in certain cases. Therefore, the fact of exceeding the acceptable residence time requirements of the system may be detected only after its maintenance. When the service requirements and check the condition of $\mathcal{\tau}_{\boldsymbol{s}} \leq \mathcal{\tau}_{\boldsymbol{s}}^{*}$ produced in different computers on the network, it is very difficult, if not impossible, process organization cessation (interruption) maintenance requirements, even if the condition is violated $\tau_{\boldsymbol{s}} \leq \mathcal{\tau}_{\boldsymbol{s}}^{*}$. (This is the case in this embodiment, ie in the service has no way of measuring time $\mathcal{\tau}_{\boldsymbol{s}}$.)

Note that, for this embodiment $N>1$, when $P_{2}$ loss do not affect the performance and service of the queue, as in any case the requirements are serviced completely.

To determine the characteristics of the system on the basis of the algorithm, the respective programs written in Pascal and bulky computing experiments conducted, and used as input data:

$$
\lambda \in(0.001,0.005,0.010,0.015,0.020)
$$

$$
\begin{aligned}
& m=10, \quad \tau_{s}^{*}=\mathbf{1 0 O}, \\
& \mu \in(0.010,0.016,0.020,0.020,0.021,0.022), \\
& \rho \in(0.1,0.3,0.5,0.7,0.9)
\end{aligned}
$$

The results obtained are shown in Tables 1-3.

$$
\begin{aligned}
& \text { The dependence of } \quad L_{q}=f(\rho) \text { and } \\
& \boldsymbol{L}_{\boldsymbol{s}}=\boldsymbol{f}(\boldsymbol{P})_{(\text {Table } 2) \text { that the maximum }}
\end{aligned}
$$
value is achieved at $L_{q}\left(L_{S}\right)$ and after these values $\boldsymbol{P}=\mathbf{0 . 3}(\mathbf{0 . 5})$, the service process is accompanied by a downward trend in the value $L_{q}\left(L_{S}\right)$

Table 1. $L_{q}\left(L_{S}\right)$

\begin{tabular}{|l|l|l|}
\hline $\boldsymbol{\rho}$ & $\boldsymbol{L}_{q}$ & $\boldsymbol{L}_{\boldsymbol{s}}$ \\
\hline O. & $1.05266315789504161 \mathrm{E}-11$ & 0.10000000000105263 \\
& & 2 \\
\hline
\end{tabular}




\begin{tabular}{|l|l|l|}
\hline O. & $3.3347753832124043 \mathrm{E}-10$ & 0.3125000003334775 \\
\hline O. & $2.004626871380261 \mathrm{E}-10$ & 0.5000000002004626 \\
\hline O. & $1.956307260232261 \mathrm{E}-10$ & $\begin{array}{l}4.4995066983534367 \mathrm{E}- \\
10\end{array}$ \\
\hline O. & $1.0995045078373537 \mathrm{E}-10$ & $\begin{array}{l}2.4738851425784085 \mathrm{E}- \\
10\end{array}$ \\
\hline
\end{tabular}

In Table 3, data are obtained depending $\tau_{q}=f(\lambda)$

$\tau_{s}=f(\lambda)$. With increasing values of

$\lambda$ and $\mathcal{\tau}_{s}$ decreases the value of the optimal value of the characteristics of the system obtained by $\lambda=0.020$.

Table 2. $\tau_{s}^{*}=100$

\begin{tabular}{|c|c|c|}
\hline$\lambda$ & $\mathcal{T}_{q}$ & $\mathcal{\tau}_{s}$ \\
\hline 0.001 & $\begin{array}{l}1.052631578950416 \\
\text { E-8 }\end{array}$ & 100.00000001052632 \\
\hline 0.005 & $\begin{array}{l}6.669550766424808 \\
\text { E-8 }\end{array}$ & 62.500000066695506 \\
\hline 0.010 & $\begin{array}{l}2.004626871380261 \\
\text { E-8 }\end{array}$ & 50.000000020046265 \\
\hline 0.015 & $\begin{array}{l}1.304204840154840 \\
7 \mathrm{E}-8\end{array}$ & 47.619047633208966 \\
\hline 0.020 & $\begin{array}{l}5.497522539186769 \\
\text { E-9 }\end{array}$ & 45.45454546004298 \\
\hline
\end{tabular}

It should be noted that these results are improved with increasing values of $N$ (Table 3), and the total loss of information reach its minimum at $N=4,5$.

Table 3. $m=10, \tau_{s}^{*}=1 \mathrm{OO}$

\begin{tabular}{|l|l|l|l|}
\hline $\boldsymbol{N}$ & $\boldsymbol{P}_{\mathbf{1}}$ & $\boldsymbol{P}_{2}$ & $\boldsymbol{P}$ \\
\hline 2 & $9.003893535306852 \mathrm{E}$ & 9.00389353404013 & \\
& -10 & $\mathrm{E}-10$ & 1.80077870 \\
& & & $69346985-9$ \\
\hline
\end{tabular}

\begin{tabular}{|l|l|l|l|}
\hline 3 & $5.011567178659951 \mathrm{E}$ & $\begin{array}{l}5.011567178241353 \\
\mathrm{E}-10\end{array}$ & $\begin{array}{l}1.00231343 \\
56901306 \mathrm{E} \\
-9\end{array}$ \\
& & & \\
\hline 4 & $4.499506698714965 \mathrm{E}$ & 4.499506698353436 & \\
& -10 & $\mathrm{E}-10$ & 8.99901337 \\
& & & $068401 \mathrm{E}-$ \\
& & & 10 \\
\hline 5 & $2.473885142689683 \mathrm{E}$ & 2.473885142578408 & \\
& -10 & $5 \mathrm{E}-10$ & 4.94777028 \\
& & & $526809 \mathrm{E}-$ \\
& & & 10 \\
\hline
\end{tabular}

As already noted, in order to confirm the adequacy of the analytical (A) model of the language GPSS (General purpose simulation system) developed models (I), run the simulation model with the following results [5-6]. GPSS World Simulation Report - Untitled Model 4.1.1

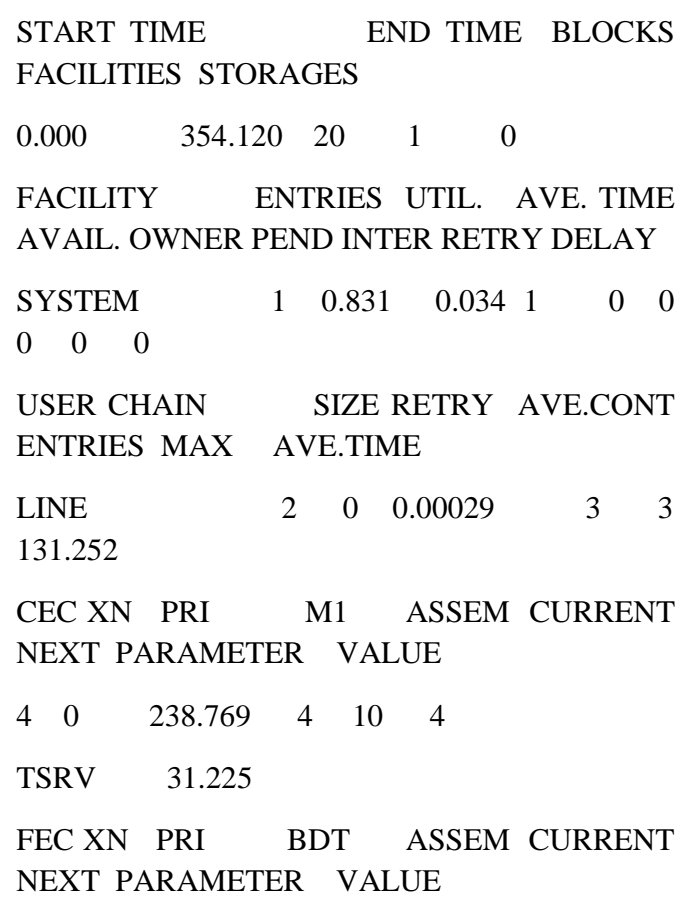

$\begin{array}{lllll}5 & 0 & 379.643 \quad 5 & 0\end{array}$,

\section{CONCLUTION}

This paper analyzes the results of analytical and simulation models of distributed networks with dynamic priorities and a limited buffer residence time requirements of the network is limited. The network constraint violation results in a loss requirements. Comparative analysis of the results of analytical and simulation methods for the problem of minimizing the loss of claims due to the preventive removal of some of them, without waiting for the service indicate the adequacy of the analytical results. These models cover a wide class of advanced networks for different purposes. It is shown that the length of this class is always advisable to forcibly remove some of the requirements of the waiting queue, thereby 
reducing the load on the servicing devices , and ultimately minimize the total losses of both kinds.

\section{REFERENCES}

[1] Kleinrock L. queuing theory. M $\quad$. Mechanical Engineering, 1979.1997.

[2] Pitts J.M., Schormans J.A. Introduction to ATM design and performance.NY: John Wiley \& Sons., 1997.

[3] Communication seti.- Kleinrock L. M .: Science, 1970, c.256..-pp.1184-1189.

[4] Mammadli EM, Slipchenko AI, Husidman V.B.Modeli organization dispatch many CPU computing real-time systems. Automation and Remote Control, M.: 1991s.167-175.vol. 138. - pp.7-29.

[5] Ismaylov BG, FA Dadgar Analysis of the characteristics of the networks with dynamic priorities and a limited buffer. Information tehnologiimodelirovaniya and management. Voronezh, №3 (81) 2013 s.292-296.
[6] Kelton W. Lowe A.Imitatsionnoe simulation. Classic S.S. The 3rd edition of St. Petersburg .: Peter, Kiev: BHV Publishing Group, 2004 to -847.

[7] Kim Ji Song, A.Z.Melikov, BG Ismailov Optimal dynamic assignment of priorities in queuing systems with two types of applications and Riga. AVT №6,2008, s5765 .

[8] Chao H.J., Peckan I.H. Queue management with multiple delay and loss priorities for ATM switches // Proc. ICC'94,1994.-pp.1184-1189.

[9] Ismayilov BG design a distributed network of service production facilities and gas treatment // $\mathrm{M}$. Gas promyshlennost.NTS. 2001, №1. s.29-35.

[10] Communication seti.- Kleinrock L. M .: Science, 1970. 